ENCYCLOPÉDIE Encyclopédie berbère

BERBERE

32 | 2010

32 | Mgild - Mzab

\title{
Musiques berbères du Maroc
}

M. Rovsing Olsen

\section{OpenEdition}

Journals

Édition électronique

URL : https://journals.openedition.org/encyclopedieberbere/662

DOI : 10.4000/encyclopedieberbere.662

ISSN : 2262-7197

\section{Éditeur}

Peeters Publishers

\section{Édition imprimée}

Date de publication : 31 décembre 2010

Pagination : $5121-5130$

ISBN : 978-90-429-2369-0

ISSN : 1015-7344

\section{Référence électronique}

M. Rovsing Olsen, « Musiques berbères du Maroc », Encyclopédie berbère [En ligne], 32 | 2010 , document M146, mis en ligne le 11 novembre 2020, consulté le 17 février 2022. URL : http:// journals.openedition.org/encyclopedieberbere/662 ; DOI : https://doi.org/10.4000/ encyclopedieberbere.662

Ce document a été généré automatiquement le 17 février 2022.

(c) Tous droits réservés 


\section{Musiques berbères du Maroc}

\section{Rovsing Olsen}

1 Le Maroc peut se prévaloir d'une très grande richesse de ses pratiques musicales. Celles des Berbères y sont particulièrement vivaces. Des festivals ont pu contribuer à faire connaître un certain nombre d'entre elles, mais c'est dans leur milieu naturel qu'elles prennent tout leur sens. Elles s'insèrent étroitement dans les techniques agricoles et contribuent à leur efficacité. Les musiciens sont aussi des agriculteurs, et c'est tout naturellement qu'ils puisent parfois dans l'agriculture des structurations et des thématiques pour leurs musiques. Musique et agriculture ont en commun des fonctionnements sociaux et des concepts. Il n'est donc pas surprenant que dans la musique, parallèlement à un processus agricole annuel, un effort de reconstruction se manifeste chez les Berbères d'année en année en vue d'une certaine permanence.

Dans leur musique, qui inclue généralement la poésie et le mouvement ou la danse, chaque groupe tribal a ses expressions musicales propres qui le démarquent de celles de ses voisins, contribuant ainsi à la diversité si remarquable au Maroc. Certaines grandes aires stylistiques musicales peuvent malgré tout être observées: elles recouvrent dans l'ensemble les aires linguistiques tarifit, tamazizt et tašlhiyt des différentes régions berbérophones (Rif ; Moyen Atlas et Haut Atlas oriental ; Haut Atlas central et occidental, Anti-Atlas et plaines du Sous). Même si l'on est loin d'avoir étudié l'ensemble des traditions musicales berbères (le Rif en particulier reste peu connu pour sa musique), on peut noter comme trait pertinent général l'importance accordée à huit éléments caractéristiques :

1. le registre chanté aigu (en particulier chez les hommes) et le caractère projeté de la voix ;

2. les grands chœurs (dansants ou actants) à l'unisson ou en hétérophonie ;

3. les modalités d'exécution en alternance (antiphonale entre deux chœurs, ou responsoriale entre solistes et chœurs) y compris sur le plan poétique avec l'omniprésence de forme dialoguées ou de joutes oratoires chantées ;

4. la mise en espace sonore ;

5. des procédés de structuration, propres à chacun des types de répertoires, communs aux composantes mélodique, poétique, rythmique et chorégraphique ; 
6. le tambour sur cadre et des cycles polyrythmiques impliquant une hiérarchie entre les musiciens ;

7. des temporalités (suites de chants ou de musiques chantées et dansées) devant être appréhendées souvent sur de longues périodes (plusieurs heures, voire plusieurs jours dans certains rituels) ;

8. une poésie orale chantée construite à partir de formules matricielles composées de monosyllabes (la, lay, da, lal, etc.) et explicitées dans la performance improvisée comme un refrain initial avant les vers signifiants; ces formules ou «vers vides » déterminent en particulier le nombre et la nature des syllabes des vers (Jouad 1995).

3 Les points de divergence sont liés à des clivages régionaux qui portent surtout sur le langage musical, par exemple des mélodies plutôt pentatoniques et parfois à grands sauts chez les Chleuhs; des mélodies à ambitus restreint (souvent chromatiques), bicordales, tricordales, tétracordales ou pentacordales chez les Berabers.

Quelles que soient les régions, on peut distinguer les chants rituels (agricoles ou de mariages), qui concernent seulement certaines parties de la population, des musiques de fête qui impliquent l'ensemble des communautés; lieux importants de créativité poétique, les fêtes encadrent de nombreux types de chants dansés qui par la suite pourront être repris par des hommes comme par des femmes, ou échangés à distance entre eux, au cours d'activités solitaires dans des situations du quotidien le plus souvent extérieures. D'autres musiques chantées soutiennent des travaux d'entraide, des situations de rencontres ou d'accueil d'invités (y compris rituelles) en différentes circonstances, souvent dans une certaine effervescence. À ce titre, ces musiques pourraient être qualifiées de "chants de compagnie », selon l'expression de Bernard Lortat-Jacob (à paraître) à propos de chants équivalents répandus dans faire méditerranéenne.

5 Partout chez les Berbères, la musique intervient surtout dans les passages : en fin ou en début de cycles qu'ils soient humains (la jeune fille atteignant l'âge du mariage, le garçon celui de la circoncision, le vieillard celui de la mort), végétaux (dépiquage et vannage de l'orge; fécondation du dattier; labours) animaux (avant le sacrifice dans certains rites), ou climatiques (gel, canicule). Cette conception de la place de la musique va de pair avec une gestion collective de l'ensemble des activités musicales aussi bien qu'agricoles, rituelles, artisanales, etc., permettant la mobilisation de tous au même moment sous formes de systèmes d'entraide ou de tours de rôle entre familles.

6 Signalons l'existence d'une tradition de musiques de bardes semi-professionnels ou professionnels qui n'a pas cessé de prendre de l'importance au cours du $20^{\mathrm{e}}$ siècle. Issues des traditions montagnardes, ces musiques font intervenir des instruments à cordes.

\section{Chants rituels}

7 On distingue chez les Berbères d'importants répertoires de chants rituels propres à chaque tribu: de tels répertoires sont exécutés, d'une part lors de certaines activités agricoles, d'autre part au cours de certaines parties du mariage s'adressant à la mariée. Dans un cas comme dans l'autre, les différents chants se succèdent selon un ordre prescrit qui se réfère à la transformation de la céréale ou de la jeune fille. 
8 Pour le dépiquage et le vannage de l'orge (et du blé), l'ensemble du processus d'épuration de la céréale jusqu'à sa forme finale de grain est soutenu par des chants spécifiques dits ladkar dans l'Anti-Atlas et le Haut Atlas occidental. Le mode de fonctionnement de ces activités est discret: quelques hommes se retrouvent tôt le matin sur l'aire à battre (chaque famille a son aire qu'elle partage quelquefois avec d'autres) et chantent sur le mode responsorial (solo et chœur) tandis que les animaux (ânes, mulets), attachés à un piquet planté au milieu de l'aire, tournent pendant des heures pour piétiner les gerbes et ainsi séparer le grain de l'épi. Chacune des étapes du dépiquage possède son propre chant. Le premier, qui s'adresse aux animaux, est emblématique : son refrain chanté en recto tono (sur une même hauteur) est construit sur des mots du type ya ya uhu yallahu et donne parfois son nom à l'ensemble, (ihiwayahu, par exemple). Faisant suite au dépiquage, le vannage se déroule, quant à lui, en famille : hommes, femmes, jeunes et vieux disposés en deux rangs face à face autour du tas de céréales chantent en antiphonie tout en jetant en l'air le mélange issu du dépiquage à l'aide de fourches à trois dents afin de séparer le grain de la balle et de la paille. Le vent propice n'est pas continu, et les vanneurs doivent attendre parfois de longs moments qu'il souffle, d'où un déroulement acté et chanté entrecoupé par de longues pauses. Dans un mélange de berbère et d'arabe, les textes des chants, à caractère religieux que ce soit pour le dépiquage ou pour le vannage, font de nombreuses allusions à la mort et au jugement dernier. Pendant cette période, dite des aires à battre, il arrive qu'il y aient plusieurs dépiquages ou vannages simultanés sur différents aires en même temps ; même s'ils suivent chacun leur propre temporalité (en fonction du début des travaux et de la quantité de céréales), ils illustrent alors de manière remarquable, par des pratiques chantées similaires, le vécu commun de cette période d'activités partagées, quoique fractionnées sur plusieurs lieux.

Dans le Moyen Atlas et le Haut Atlas oriental, les chants religieux ahellil [ihellill] semblent jouer un rôle équivalent aux ladkar des Chleuhs même si des témoignages déjà anciens (Chottin 1939 :22) attestent d'une application nettement plus étendue avec des thématiques qui sortent du champs strictement religieux : ils sont chantés par des hommes et des femmes non seulement lors de nombreuses activités agricoles mais aussi au cours de rites du cycle de la vie ainsi que lors de travaux du quotidien (mouture du grain par exemple) (Taifi $1994: 140$ ).

Pour les mariages, les chants rituels (qu'il faut distinguer des simples chants de fête) sont exécutés a cappella par les femmes adultes (tifqqirin). Chaque groupe tribal a son propre répertoire de mélodies (nommé asnimmr, urrahman, izwirrign, asallaw, urar, etc. selon les tribus). Ordonnés en une douzaine de suites de différentes longueurs, correspondant à autant d'étapes, les chants portent le rituel de bout en bout : par leur caractère stylistique (différent dans chaque tribu), ces mélodies, qui paraissent comme des variantes les unes des autres, constituent un fil conducteur permettant de saisir et de délimiter le rituel au sein des nombreuses autres activités et festivités du mariage. La pratique antiphonale sur des lieux sans cesse renouvelés pour chaque mélodie chantée permet un jeu sur l'espace avec de nombreuses dispositions variées : un chœur sur le toit, un autre devant la porte; un chœur dans la chambre de la mariée, un autre devant la chambre, etc. Les femmes chantent en effectuant des actes précis : cérémonie du tri du grain, réception des garçons d'honneur, habillage de la mariée, coiffure de la mariée, apport et exposition du trousseau, cérémonie du puits, cortèges, etc. ; de courte durée, réparties sur une semaine, certaines séquences rituelles sont chantées à l'aube, 
d'autres la nuit, d'autres encore dans la journée. Elles sont toujours initiées par trois youyous et par un chant d'exorde. De nombreuses lamentations sont de plus émises tout au long du rituel, tantôt par des femmes, tantôt par la mariée.

11 Pour la circoncision, les chants rituels seraient du même type que ceux destinés aux mariages excepté en ce qui concerne les textes (voir par exemple Lortat-Jacob $1980: 57$ et 83-84).

\section{Musiques de fêtes}

12 Les pratiques musicales les plus fédératrices sont celles qui rassemblent les hommes et les femmes dans des performances chantées et dansées à l'occasion des fêtes importantes (y compris celles des mariages). Soutenus par des cycles polyrythmiques joués aux tambours sur cadre et motivés par les joutes poétiques chantées, des grands chœurs antiphonaux évoluent selon des chorégraphies et des figures précises indiquées par des chefs de danse. Le côté festif et spectaculaire (les protagonistes sont dans leurs plus beaux vêtements) ne doit pas faire perdre de vue la valeur didactique de ces évènements qui inculquent à la fois des règles de comportement, des attitudes du corps et un art élaboré de la parole. Ces pratiques qui ont lieu en plein air, de la nuit jusqu'au lever du soleil, se déroulent en présence de l'ensemble du village (ou du campement) et de ses invités, venus parfois en très grand nombre. Cet auditoire large et très attentif, regroupé selon l'âge, le sexe et parfois le statut social (il arrive que les invités d'honneur soient placés au milieu des chanteurs-danseurs), réagit selon des codes établis : youyous des femmes, appels ou coups de fusils tirés en l'air par des hommes, chants de compagnie. Selon les régions, les musiques chantées et dansées se manifestent principalement sous deux formes différentes: l'ahidus [haidus] dans la région tamaziðt, et l'aḥwaš dans la région tašlhiyt, deux termes qui recouvrent également les fêtes au cours desquelles se déroulent ces pratiques.

13 L'ahidus. Selon les groupes tribaux, les femmes et les hommes se disposent dans l'espace en cercle ou en ligne, soit côte à côte, soit vis à vis, en deux rangées séparées toujours face au centre. Seuls les hommes jouent du tambour, et ils sont aussi seuls à chanter dans la plupart des cas attestés. Chez les Ayt Mguild, le cercle se compose d'hommes et de femmes en alternance qui évolue sur une formule rythmique tambourinée asymétrique à cinq temps (répartis sur deux hauteurs grave et aiguë), le mouvement général se fait ondulant avec une gestuelle spécifique. Faisant suite aux izlan (sing.izli) échangés entre poètes-chanteurs, les double chœurs masculins chantent à l'unisson, parfois en hétérophonie, par exemple à la tierce (Chottin 1939 : 16-21). Chez les Ichkern, comme chez les Ayt Mguild (ibid. : 22) les izlan peuvent être chantés aussi sous formes d'énigmes. Echangées entre poètes-chanteurs, alors que l'ahidus continue, ces énigmes mettent à l'épreuve la capacité des solistes à répondre rapidement, et cela sur la même mélodie et le même vers que leur « partenaire » (Guennoun 1991 :125).

Partout, différents types d'ahidus peuvent être observés : par exemple, chez les Ayt Mguild, deux ahidus se singularisent en fonction de la présence ou non de battements de mains ou de pieds : tamhawšt et tamseralit. (Chottin 1939: 21). Chez les Ayt Yahya, deux autres types d'ahidus peuvent être reconnus à Tounfite : le hayfa ou tayzzaft (« la longue », en référence à la phrase mélodique et aux modalités d'exécution), le plus ancien, sans participation aucune des femmes, évolue comme chez les Ayt Mguild sur un rythme à cinq temps, et se danse avec une grande retenue en deux lignes ou « ailes » 
se faisant face ; l'amssad, quant à lui, évolue sur des contrastes d'intensité et de tempo ; des jeunes entremêlés de femmes le chantent et le dansent (Lortat-Jacob 1994 : 52-53). Chez les Ayt Haddidou, où l'ahidus se danse en deux lignes parallèles d'hommes et de femmes qui se font face, le pendant féminin (chanté par les femmes exclusivement) est appelé tahidust (Kasriel 1989: 130-131). La poésie chantée est ici dénommée plutôt tamawayt ou lmayt qu'izli. Le cycle rythmique à cinq temps paraît une caractéristique importante de l'ahidus. Certains chercheurs mentionnent des cycles à dix temps divisibles en deux "mesures» différentes de cinq temps (Karpati 1961: 456-457). D'autres rappellent aussi l'existence de différentes phases rythmiques liées au tempo: l'un modéré (binaire), l'autre rapide (binaire ou ternaire) (Aydoun 1992 : 84-85).

L'aḥwaš. On connaît sous le terme général d'aḥwaš de nombreuses traditions du Haut Atlas central et occidental, de la plaine du Sous et de l'Anti-Atlas. L'ahwaš évolue pendant la nuit par phases d'une quinzaine de minutes chacune, au cours desquelles se mettent en place de manière progressive, et sur fond de transformation, les éléments constitutifs de la mélodie et du rythme. Chez les Ayt Mgoun du Haut Atlas central, l'ahwaš comporte cinq phases : $1^{\circ}$ solo masculin sur un distique répété deux ou trois fois ; $2^{\circ}$ reprise répétée de ces deux vers par le chœur masculin (le deuxième vers) puis féminin (le premier vers) en alternance; $3^{\circ}$ intervention des tambours sur cadre (jusqu'à une trentaine) et de la danse des chœurs qui continuent à chanter; transformation graduelle de la formule rythmique qui passe de quatre temps à trois temps inégaux et accélération progressive régissant la "montée » recherchée. $4^{\circ}$ continuation de la «montée» et intervention de chants masculins en solo (tizrrarin, sing. tazrrart) annoncés par un long cri ahayyu qui fait taire les chœurs; $5^{\circ}$ formule conclusive des tambours (Lortat-Jacob 1980 : 65-68). La joute initiale lmsaq est chantée par un soliste auquel répondent en alternance les chœurs d'hommes et de femmes. On notera ici l'efficacité du mouvement effectué par les musiciens jouant au tambour sur cadre afin de parvenir à ponctuer à intervalles espacés, comme un seul homme, le chant non mesuré du soliste.

Dans l'Anti-Atlas, une distinction domine entre ahwaš dit "des femmes " (aḥwaš $n$ tmyarin) ou agwal (chez les Isaffen) et pratiques masculines appelées ddrst (chez les Ida Ouzddout) ou ahnaqqar, toutes centrées sur les joutes poétiques: la première partie de la nuit est consacrée aux pratiques masculines, la seconde à celles des femmes. Dans leurs aḥwaš, les hommes sont généralement disposés en une longue rangée au sein de laquelle les poètes tiennent un tambour sur cadre tallunt. Ils cachent leur visage derrière cet instrument en chantant. À tour de rôle, les poètes-chanteurs improvisent quelques vers sur une thématique d'actualité ; d'une portée plus générale, le dernier est repris par la rangée en double chœur alterné jusqu'à l'intervention du poète suivant. En guise de conclusion, la rangée se ferme et entame une danse en ronde sur le dernier vers chanté. Cette danse se poursuit jusqu'à l'interruption. Un nouvel échange se met alors en place, et ainsi de suite pendant plusieurs heures. Les mouvements de danse accomplis face au centre sont souvent vigoureux avec des sauts, des mouvements de tête et de bras, etc. Dans l'Anti-Atlas, le gros tambour à deux peaux ganga et les cliquettes tiqrqqawin font partie de l'instrumentarium de tout aḥwaš masculin et féminin aux côtés des tambours sur cadre.

Dans l'ahwaš «des femmes », la joute se déroule entre un poète et une poétesse. Un poète-chanteur s'introduit au milieu des deux rangées en demi-cercles de femmes se faisant face : il chante plusieurs vers aboutissant à un vers à portée générale avant de 
reprendre sa place au milieu des auditeurs. Les femmes reprennent le dernier vers en alternance avant d'entamer leur réponse dont les vers sont soufflés au fur et mesure par la poétesse située soit au milieu de l'un des chœurs, soit derrière, dans l'obscurité. Tout en chantant, les femmes effectuent une rotation : elles sont accompagnées par les tambours des hommes sur un cycle qui se présente souvent sous la forme de cinq frappes inégales. L'intervention de la poétesse est latente, puisque ce sont les chœurs qui chantent ses paroles improvisées. Les deux chœurs dans l'aḥwaš opèrent des segmentations variées dans la mélodie et dans les vers échangés et le processus d'épaississement sonore culmine avec l'intervention des chants de compagnie responsoriaux tazrrart par des groupes successifs du public masculin.

Dans le Haut Atlas occidental, c'est un ạ̣waš instrumental, la musique dansée "guerrière " taskiwin, qui domine. Evoluant selon des figures de danse précises, les danseurs s'accompagnent d'un petit tambour de poterie en forme de gobelet agwal, formant ainsi avec les trois joueurs de tambours sur cadre tallunt la partie rythmique grave de différents cycles polyrythmiques. La partie mélodique est assumée par des flûtes talcwwatt. Chaque danseur porte un poignard dans un fourreau en argent qui pend en bandoulière, et à l'épaule gauche une corne à poudre richement décorée, à laquelle sont attachés des grelots que les danseurs font sonner par le tremblement de l'épaule gauche dans la partie "secouée » de la taskiwin. C'est cette corne à poudre (tiskt, plur. taskiwin) qui donne son nom à la danse. Le déroulement suit un parcours précis : celui des Ayt Tajelt par exemple comprend huit taskiwin qui se différencient par leurs parties initiales (évoquant des mouvements et des attitudes guerrières variés) et se rapprochent par leur partie finale assus (« secousse »). Les taskiwin sont entrecoupées d'intermèdes souvent humoristiques et de danses des jeunes filles auquels participent les musiciens à la flûte et aux tambours sur cadre et dans lesquelles le mouvement des épaules est central (d'où le nom izariwn, « épaules »).

\section{Musiques des bardes}

Les Berbères du Maroc ont aussi une longue tradition de musiciens semi-professionnels ou professionnels, petits ensembles de bardes rwyns ou imdyazn [imlyazn] qui tiraient traditionnellement un complément de subsistance en parcourant la montagne dans l'arrière-saison. Encourageant les hommes vaillants, dans les conflits entre tribus ou dans les luttes pour l'indépendance, analysant les événements politiques nationaux ou internationaux, commentant les faits et attitudes des hommes émigrés ainsi que leur religion ou leurs mœurs, les bardes ont joué jusqu'à nos jours un rôle culturel non négligeable. Ils sont de surcroît redoutés par ceux qui font l'objet de leurs railleries. Leurs performances constituent de véritables spectacles avec danse, bouffonnerie, dialogues avec les spectateurs, et souvent quête finale.

Les imdyazn, originaires du Haut Atlas central et oriental, se composent de quatre personnes : un chef (amdyaz), deux « répondants » ou « accompagnateurs » (irddadn) qui reprennent les vers de l'amdyaz en alternance avec lui et jouent du tambour sur cadre allun ou du lkamanja (alto ou violon joué à la verticale, parfois aussi par l'amdyaz), et le bu uranim («celui du roseau»), généralement un noir, qui chante ou joue un instrument à vent (clarinette double ayanim); paré et dansant comme une femme, il joue le rôle d'un bouffon et dialogue avec les spectateurs (Lortat-jacob 1980 :43-48). Leur chant est de deux sortes : timdyazin (sing. tamdyazt) et izlan. Alors que les izlan sont 
plutôt chantés par les irddadn, les tamdyazt sont chantées et composées par les bardes. D'autres ensembles se produisent dans les bourgades. À titre d'exemple, les chanteusesdanseuses šixat jouant aux tambours et dont les izlan sont centrés sur des thèmes légers; elles se font accompagner au lkamanja ou au luth (luțar ou lginbri) par des hommes šix. (Lortat-jacob 1994 :54-55).

21 Les rrwways. Ces ensembles itinérants chleuhs, sont constitués, quant à eux, principalement d'un poète-chanteur rrrays, qui s'accompagne à la vièle monocorde rribab, de plusieurs joueurs de luth à manche long munis de trois ou quatre cordes (luțar ou lginbri) et d'un joueur de cloche naqus. S'y joignent parfois des chanteuses-danseuses rraysat jouant des cymbalettes nuiqsat fixées sur chacune des deux mains. Avec le développement du disque et du spectacle en milieu urbain, certaines rrraysat ont créé avec succès leurs propres ensembles. La performance traditionnelle des rrrways se déroule selon une suite de segments à caractères chanté, dansé, instrumental ou bouffon, choisis en fonction de l'auditoire. La poésie chantée (amarg) est toujours le segment le plus attendu (Chottin 1933 et 1939 et Schuyler 1984). Très populaire en milieu berbère, ce type de poésie chantée a aussi fait l'objet de nombreux recueils et d'analyses portant sur la poésie et sur sa métrique.

\section{BIBLIOGRAPHIE}

AYDoun A., Musiques du Maroc, Eddif, Casablanca, 1992.

CHоттіN A., Corpus de musique marocaine II. Musique et danses berbères du pays chleuh, Heugel, Paris, 1933. [Rééd. Librairie Libre-Service, Casablanca, 1987].

CHоттіN A., Tableau de la Musique Marocaine, Paul Geuthner, Paris, 1939. (Rééd. Société nouvelle Librairie Orientaliste Paul Geuthner, Paris, 1999).

DeLl F. et ELMEDLAoui M., Poetic Meter and Musical Form in Tashlhiyt Berber Songs, (avec un CD Rom), Series : Berber Studies, vol. 19, Rüdiger Köppe Verlag, 2008.

GALAND-PERNET P., Recueil de poèmes chleuhs 1 : chants de trouveurs, Klincksieck, Paris, 1972.

GUENNOUN S., « Littérature des Berbères de la Haute Moulouya », Etudes et Documents berbères 8, p. 1 13-134, 1991.

HOFFMAN K.E., « Generational Change in Berber Women's Song of the Anti-Atlas Mountains, Morocco », Ethnomusicology 46, nº 3, p. 510-540, 2002.

JOUAD H. et LORTAT-JACOB B., La saison des fêtes dans une vallée du Haut-Atlas, Le Seuil, Paris, 1978.

JOUAD H. et LORTAT-JACOB B., « Les modèles métriques dans la poésie de tradition orale et leur traitement musical ", Revue de musicologie 68 n 1-2, p. 174-197, 1982.

JOUAD H., « Les tribulations d'un lettré en pays chleuh », Études et Documents Berbères 2, p. 27-41, 1987. 
JOUAD H., « La matrice rythmique. Fondement caché du vers », Études et Documents Berbères 3, p. $47-59,1987$.

JOUAD H., Le calcul inconscient de l'improvisation : Poésie berbère, rythme, nombre, et sens, Peeters, ParisLouvain, 1995.

KARPATI J., « Mélodie, vers et structure strophique dans la musique berbère (imazighen) du Maroc Central », Studia Musicologica 1, n 3-4, p. 451-473, 1961.

KASRIEL M., Libres femmes du Haut-Atlas? Dynamique d'une micro-société au Maroc, L'Harmattan, Paris, 1989.

LAOUST, É., Noces berbères. Les cérémonies du mariage au Maroc. Edition établie par Claude Lefébure, Edisud/La Boîte à Documents, Aix-en-Provence, 1993.

LEFÉBURE C., « Contrat mensonger. Un chant d'amdyaz sur l'émigration », Études et Documents Berbères 3, p. 28-46, 1987.

LORTAT-JACOB B., "Music as a Collective Enterprise : The Case of Berber Music of the High Atlas ", World of Music 3, p. 62-76, 1979.

LORTAT-JACOB B., Musique et fêtes au Haut-Atlas, Mouton-EHESS, Paris, 1980.

LORTAT-JACOB B., "Community Music as an Obstacle to Professionalism : a Berber Example", Ethnomusicology XXV nº 1, p. 87-98, 1981.

LORTAT-JACOB B., "Music and Complex Societies : Control and Management of Musical Production", Yearbook for Traditional Music XVI, p. 19-33, 1984.

LORTAT-JACOB B., Musiques en fête. Maroc, Sardaigne, Roumanie, Société d'ethnologie (« Hommes et Musiques »), Nanterre, 1994.

LORTAT-JACOB B., "Singing in Company". Multipart singing in the Mediterranean Area”, Vienne [sous presse].

OULD-BRAHAM O., « Voyages scientifiques de Boulifa (Maroc, 1905 ; Kabylie, 1909-1912) », Études et Documents berbères 13, p. 27-78, 1995.

PARIS A., « Haouach à Télouet », Hespéris 1, p. 209-216, 1921.

PEYRIGUERE J., « Poésie et danse dans une tribu berbère du Moyen-Atlas », Études et Documents Berbères 15-16, p. 219-248, 1998. [Article paru dans le Maroc catholique en cinq livraisons (de janvier 1934 à mars 1934) sous le pseudonyme de Paul Hector].

PEYRON M., "Isaffn ghbbanin (rivières profondes)". Poésies du Moyen Atlas marocain traduites et annotées, Wallada, Casablanca, 1993.

PEYRON M., « Danse », Encyclopédie Berbère XIV, p. 2204-2213, 1994.

ROVSING OLSEN M., Chants de mariage de l'Atlas marocain, thèse de doctorat de troisième cycle (directeur Gilbert Rouget), Unversité Paris X-Nanterre, 1984.

Rovsing olsen M., Chants et danses de l'Atlas (Maroc). Preface par Bernard Lortat-Jacob), Cité de la musique/Actes Sud, Arles, 1997. (1 CD inclus).

ROVSING OLSEN M., "Modalités d'organisation du chant berbère : paroles et musique ", Journal of Mediterranean Studies 6, $\mathrm{n}^{\circ}$ 1, p. 88-108, 1996.

ROVSING OLSEN M., « Le musical et le végétal : essai de décryptage. Exemple berbère de l'AntiAtlas ", L'Homme. Revue française d'anthropologie 171-172, p. 103-124, 2004. 
RouX A., « Les imdyazen ou aèdes berbères du groupe linguistique beraber ", Hespéris VIII n ${ }^{\circ}$ 3-4, p. 231-251, 1928.

SCHUYLER P. D., A Repertory of Ideas : the Music of the Rwais, Berber Professional Musicians from Southwestern Morocco, Ph. D, University of Washington, 1979.

SCHUYLER P. D., « Rwais and Ahwash : Opposing Tendencies in Moroccan Berber Music and Society ", The World of Music 21, $\mathrm{n}^{\circ}$ 1, p. 65-80, 1979.

SCHUYLER P. D., "The Rwais and the Zawia : Professional Musicians and the Rural Religious Elite in Southwestern Morocco", Asian Music XVIII n 1, p. 114-131, 1985.

TAÏFI M., « La transcription de la poésie orale : de la transparence orale à l'opacité scripturale », Études et Documents Berbères 11, p. 133-147, 1994.

INDEX

Mots-clés : Agriculture, Chant, Chleuh, Ethnologie, Ethnographie, Littérature, Maroc, Poésie 